\title{
Comparison among Three Different Steroid Therapies for Idiopathic Sudden Sensorineural Hearing Loss
}

\author{
Da Jung Jung ${ }^{\mathbb{D}}$, JiHoon Lee, Myung Hoon Yoo ${ }^{\mathbb{D}}$, and Kyu-Yup Lee $\mathbb{D}^{\mathbb{D}}$ \\ Department of Otorhinolaryngology-Head and Neck Surgery, School of Medicine, Kyungpook National University, Daegu, Korea
}

\author{
돌발성 난청에 대한 세 가지 다른 스테로이드 요법의 비교 \\ 정다정 · 이지훈 · 유명훈 · 이규엽 \\ 경북대학교 의과대학 이비인후-두경부외과학교실
}

\author{
Received January 29, 2020 \\ Revised June 4, 2020 \\ Accepted June 8, 2020 \\ Address for correspondence \\ Kyu-Yup Lee, MD, PhD \\ Department of Otorhinolaryngology- \\ Head and Neck Surgery, \\ School of Medicine, \\ Kyungpook National University, \\ 130 Dongdeok-ro, Jung-gu, \\ Daegu 41944, Korea \\ Tel $+82-53-420-5785$ \\ Fax $+82-53-423-4524$ \\ E-mail kylee@knu.ac.kr
}

Background and Objectives The optimal dose or type of systemic steroid for treating idiopathic sudden sensorineural hearing loss (ISSNHL) is unclear. Herein, we compare the efficacy of three steroid treatment protocols.

Subjects and Method We reviewed the medical records of 140 adult ISSNHL patients from a tertiary medical center. The patients were divided into three groups based on their treatment regimen: Group 1 received intravenous $10 \mathrm{mg} /$ day dexamethasone combined with intratympanic (IT) steroid injection, followed by prednisolone for 5 days after discharge; Group 2 received 10 $\mathrm{mg} /$ day dexamethasone for 5 days, followed by $5 \mathrm{mg} /$ day for 5 days over a 10 -day hospitalization period; and Group 3 received $10 \mathrm{mg}$ /day dexamethasone combined with IT steroid injection during a 5 -day hospital stay, followed by $5 \mathrm{mg}$ /day dexamethasone for 5 days after discharge. The hearing thresholds were measured using an automatic audiometer at $0.5,1,2,3,4$, and 8 $\mathrm{kHz}$. Hearing recovery on Day 90 was categorized according to Siegel's criteria.

Results Univariate and multivariate analyses showed that patients in Group 3 had the lowest hearing thresholds, and the best results for speech reception threshold and speech discrimination scores. The impact of favorable thresholds in Group 3 was better among patients with a baseline average hearing threshold of $<70 \mathrm{~dB}$. Complete recovery was more likely in Group 3 than in the other groups, based on the odds ratios.

Conclusion Administration of dexamethasone-based systemic steroid combined with IT steroid injection and a relatively long hospitalization period produced the most favorable result.

Korean J Otorhinolaryngol-Head Neck Surg 2020;63(11):497-504

Key Words Dexamethasone · Intratympanic injection - Sudden sensorineural hearing loss.

\section{Introduction}

Idiopathic sudden sensorineural hearing loss (ISSNHL) is an emerging disease in the field of otology. ISSNHL is associated with permanent hearing loss without timely and appropriate treatment, which leads to a profound decrease in the quality of life. It has been previously reported that the

This is an Open Access article distributed under the terms of the Creative Commons Attribution Non-Commercial License (https://creativecommons.org/licenses/by-nc/4.0) which permits unrestricted non-commercial use, distribution, and reproduction in any medium, provided the original work is properly cited. prevalence of ISSNHL in Korea and USA was approximately 10 per 100000 persons and 5-27 per 100000 persons, respectively. ${ }^{1-4)}$ Previous studies have reported viral infection, poor blood supply in the cochlea, or endolymphatic hydrops as associated symptoms, but the pathogenesis of ISSNHL has not yet been exactly defined. ${ }^{5)}$ The precise mechanism has not yet been defined, but corticosteroid is widely used as the first therapeutic option for ISSNHL. Various protocols with regard to the route and dose of steroid administration have been introduced. 
Systemic steroid is a well-known treatment option for ISSNHL. Although a recent meta-analysis of three randomized controlled trials produced unfavorable results associated with systemic steroid administration as opposed to placebo, many clinicians have considered steroid as the first-line therapy of ISSNHL. $\left.{ }^{6}\right)$ There are inconsistent opinions regarding the optimal dose or type of systemic steroid. A recent guideline recommended prednisone dosage of $1 \mathrm{mg} / \mathrm{kg} / \mathrm{d}$ (or an equivalent drug dose) for 7-14 days, followed by gradual tapering of dosage. ${ }^{7)}$ Intratympanic (IT) steroid injection was initially administered as salvage therapy, but recent data have shown favorable outcomes of an initial combination therapy with systemic steroid. ${ }^{8)}$ Several studies have analyzed the efficacy, optimal dose, or route of steroid administration using a small number of participants and have reported contradictory outcomes. Many centers have implemented different protocols pertaining to the dose, type, or route of steroid administration. Therefore, additional studies are needed for the identification of optimal dose or type of systemic steroid as well as the efficacy of combinational therapy with IT steroid. We want to compare the efficacy of three different protocols for the type of steroid and/or route of administration on hearing recovery.

\section{Subjects and Method}

\section{Study population}

We reviewed medical records from a tertiary medical center and identified all adult patients who were diagnosed with ISSNHL between January 2012 and June 2018. ISSNHL was defined as short-term, unilateral sensorineural hearing loss ( $\geq 30 \mathrm{~dB}$ at three consecutive frequencies) that had an onset within 72 hours without any specific cause and/or conductive problems, consistent with the definition provided in a previous report. ${ }^{7)}$ We identified 172 patients who were aged $\geq 18$ years and diagnosed with ISSNHL. From those patients, 32 were excluded either because of missing data or because they could not be followed up for more than 3 months. Finally, 140 patients were included in our study. Our center followed three different protocols for ISSNHL management (Supplementary Fig. 1). We divided three groups according to the protocols performed for different periods as follows: Group 1, 2, and 3.

After December 2015, patients were hospitalized for 5 days and intravenously administered $10 \mathrm{mg}$ dexamethasone daily combined with IT steroid injection $(0.3-0.7 \mathrm{~mL}$ of 5 $\mathrm{mg} / \mathrm{mL}$ dexamethasone per IT injection) given thrice alternate days. After discharge, patients were treated with prednisolone at a concentration of $30 \mathrm{mg}$ /day for 3 days, followed by prednisolone at a concentration of $20 \mathrm{mg}$ /day for 2 days; patients treated in this manner were categorized under Group 1. Before March 2013, patients were hospitalized for 10 days and administered with intravenous $10 \mathrm{mg}$ /day dexamethasone for 5 days, followed by $5 \mathrm{mg} /$ day dexamethasone for 5 days; such patients were categorized under Group 2. Between March 2013 and December 2015, patients were administered with $10 \mathrm{mg} /$ day dexamethasone for 5 days, followed by $5 \mathrm{mg}$ /day dexamethasone for the next 5 days; they were also given IT steroid injection $(0.3-0.7 \mathrm{~mL}$ of $5 \mathrm{mg} / \mathrm{mL}$ dexamethasone per IT injection) administered thrice on alternate days. These patients were categorized as Group 3.

\section{Study variables}

Information collected from the participants during admission included the following types of clinical data: age, sex, duration from onset to treatment, presence of tinnitus, side of impairment, performance of stellate ganglion block, and hearing data.

The hearing thresholds were measured using an automatic audiometer at $0.5,1,2,3,4$, and $8 \mathrm{kHz}$. For the impaired ear, the average hearing threshold (AHT) was calculated as puretone average of the thresholds at $0.5,1,2$, and $3 \mathrm{kHz}$. In addition, speech reception threshold (SRT) and speech discrimination scores (SDS) were noted. These measurements were taken at pretreatment, and subsequently repeated at Day 10 and Day 90 after treatment initiation. Hearing recovery on Day 90 was categorized according to Siegel's criteria; complete recovery (CR; final hearing capacity of $<25 \mathrm{~dB}$ ), partial recovery (PR; final hearing capacity of 25-45 dB, with $\geq 15 \mathrm{~dB}$ decrease), slight improvement (SI; $\geq 15 \mathrm{~dB}$ decrease with a final hearing capacity of $>45 \mathrm{~dB}$ ), no improvement (NI; $<15 \mathrm{~dB}$ decrease and a final hearing capacity of $>75 \mathrm{~dB})^{9)}$

\section{Statistical analyses}

Data were analyzed using IBM SPSS version 25 (IBM Corp., Armonk, NY, USA). Categorical variables are expressed as numbers and percentages and continuous variables are expressed as means \pm standard deviations for univariate analysis and means \pm standard errors for multivariate analysis. Pearson's $\chi^{2}$ test or Fisher's exact test was used to analyze categorical variables. For continuous variables, mean 
values among the three groups were compared using a oneway analysis of variance followed by post-hoc Turkey comparisons. Logistic regression analyses were used to estimate the odds ratios and $95 \%$ confidence intervals (CIs), which were then used to determine the relationship between the groups and CR. A univariate logistic regression analysis was performed to estimate odds ratio and $95 \%$ CI of CR using one variable alone.

Multivariate analysis was adjusted for age and duration from onset to treatment, and was focused toward the identification of clinical association and significant differences among groups. Multivariate analyses were performed using an analysis of covariance or multiple logistic regression to determine the independent predictors of hearing thresholds or $\mathrm{CR}$, for which a $p$-value of $<0.05$ was considered statistically significant. The analysis of covariance was followed by an least significance difference (LSD) post-hoc test.

All procedures performed in studies involving human participants were in accordance with the ethical standards of the institutional and/or National Research Committee (Kyungpook National University Hospital Institutional Review Board and approval No. KNUH 2019-10-035) and with the 1964 Helsinki declaration and its later amendments or comparable ethical standards. The board waived the need for informed consent, since the subjects' records and information were anonymized and de-identified prior to the analysis.

\section{Results}

\section{Baseline characteristics of participants}

Baseline characteristics are shown in Table 1. Groups 1 and 2 were predominantly composed of women. The mean age was higher in Group 1 than that in the other groups. Duration from onset to treatment was longer in Group 1 than that in the others. Group 1 had the highest incidence of tinnitus. There were no significant differences in the propor- tion of male and females, performance of stellate ganglion block, and the side of ISSNHL among the three groups.

\section{Changes in hearing thresholds according to groups}

Hearing thresholds at all frequencies $(0.5,1,2,3,4$, and 8 $\mathrm{kHz}$ ) are shown in Fig. 1 and Supplementary Table 1. Univariate analysis revealed no significant differences in baseline hearing thresholds at all frequencies among the three groups. Additionally, hearing thresholds on Day 10 were similar among the three groups. On Day 90, hearing thresholds at all frequencies in Group 3 were considerably lower than those in Group 1. Moreover, on Day 90, hearing thresholds at all frequencies in Group 2 were lower than those in Group 1, but statistical significance was low. Multivariate analyses showed similar results.

For all participants, AHTs at baseline and on Day 10 were similar among the three groups, but the value on Day 90 was the lowest with statistical significance in Group 3 (Fig. 2). Subgroup analyses conducted for baseline hearing impairment showed similar trends but with weak statistical significance. Multivariate analyses revealed that AHTs in Groups 1,2 , and 3 were $75.4 \pm 4.2,71.3 \pm 3.8$, and $72.6 \pm 3.6 \mathrm{~dB}$ at baseline, $65.8 \pm 5.1,61.0 \pm 4.5$, and $54.6 \pm 4.3 \mathrm{~dB}$ on Day 10, and $58.8 \pm 4.6,55.3 \pm 4.1$, and $42.6 \pm 4.2 \mathrm{~dB}$ on Day 90 , respectively. $P$ values among the three groups at baseline and on Days 10 and 90 were $0.772,0.245$, and 0.024 , respectively. On Day 90, LSD post-hoc test after analysis of covariance showed that Group 3 had significantly lower thresholds than the other groups ( $p=0.576$ for Group 1 vs. $2, p=0.013$ for Group 1 vs. 3, and $p=0.031$ for Group 2 vs. 3).

\section{Changes in SRT and SDT according to groups}

For univariate analysis, SRT in Groups 1, 2, and 3 were $83.8 \pm 23.5,76.7 \pm 25.3$, and $77.3 \pm 27.3 \mathrm{~dB}$ at baseline $(p=$ $0.363) ; 73.3 \pm 26.4,64.3 \pm 33.4$, and $58.6 \pm 35.9 \mathrm{~dB}$ on Day 10 $(p=0.098)$; and $63.4 \pm 25.8,54.0 \pm 35.2$, and $47.4 \pm 34.6 \mathrm{~dB}$ on

Table 1. Clinical characteristics of participants according to groups

\begin{tabular}{|c|c|c|c|c|}
\hline & Group $1(n=41, \%)$ & Group $2(n=47, \%)$ & Group $3(n=52, \%)$ & p-value \\
\hline Age (years) & $57.2 \pm 15.6$ & $47.4 \pm 15.9^{*}$ & $47.5 \pm 15.9^{*}$ & 0.005 \\
\hline Sex (men) & $14(34.1)$ & $21(44.7)$ & $28(53.8)$ & 0.166 \\
\hline Duration from onset to treatment (days) & $5.5 \pm 6.4$ & $2.9 \pm 2.0^{*}$ & $3.0 \pm 2.5^{*}$ & 0.003 \\
\hline Tinnitus & $30(73.2)$ & $28(59.6)$ & $24(46.2)$ & 0.031 \\
\hline Performance of SGB & $36(87.8)$ & $42(89.4)$ & $45(86.5)$ & 0.912 \\
\hline Side (right) & $18(43.9)$ & $28(59.6)$ & $25(48.1)$ & 0.304 \\
\hline
\end{tabular}

The data are expressed as numbers (percentages) for categorical variables and means \pm standard deviations for continuous variables. The $p$-values were tested among the three groups using one-way analysis of variance for continuous variables and Pearson's $\chi^{2}$ test or the Fisher's exact test for categorical variables. $* p<0.05$ vs. Group 1. SGB: stellate ganglion block 
Day 90, respectively ( $p=0.065$ ) (Fig. 3). SDT in Groups 1,2 , and 3 were $34.8 \pm 42.0,33.2 \pm 38.5$, and $41.1 \pm 40.7 \mathrm{~dB}$ at baseline $(p=0.595) ; 58.3 \pm 39.3,48.9 \pm 44.7$, and $60.9 \pm 43.6 \mathrm{~dB}$ on Day $10(p=0.355)$; and 66.1 $\pm 38.5,52.8 \pm 43.2$, and $72.2 \pm 37.5$ $\mathrm{dB}$ on Day 90 , respectively ( $p=0.053$ ). For multivariate analysis, SRT in Groups 1, 2, and 3 were 83.7 $\pm 4.2,76.7 \pm 3.8$, and $77.4 \pm 3.6 \mathrm{~dB}$ at baseline $(p=0.427) ; 74.0 \pm 5.4,63.9 \pm 4.8$, and $58.3 \pm 4.5 \mathrm{~dB}$ on Day $10(p=0.094)$; and 63.2 $\pm 5.3,54.1 \pm 4.8$, and $47.5 \pm 4.5 \mathrm{~dB}$ on Day 90 , respectively $(p=0.095)$. SDT in Groups 1, 2, and 3 were $34.4 \pm 6.6,33.4 \pm 5.9$, and $41.2 \pm 5.6$ $\mathrm{dB}$ at baseline $(p=0.585) ; 56.9 \pm 7.0,49.6 \pm 6.3$, and $61.5 \pm 6.0$ $\mathrm{dB}$ on Day $10(p=0.382)$; and $66.5 \pm 6.6,52.7 \pm 5.9$, and $72.0 \pm$
$5.6 \mathrm{~dB}$ on Day 90, respectively ( $p=0.052$ ). On performing univariate and multivariate analyses, SRT on Days 10 and 90 was significantly lower in Group 3 than in Group 1, whereas SDT on Day 90 was significantly higher in Group 3 than in Group 2.

\section{Hearing improvement according to groups}

The number of participants with CR, PR, SI, and NI were $3(7.3 \%), 11(26.8 \%), 11(26.8 \%)$, and $16(39.0 \%)$ in Group 1; $11(23.4 \%), 9(19.1 \%), 9(19.1 \%)$, and $18(38.3 \%)$ in Group 2; and $19(36.5 \%), 8(15.4 \%), 9(17.3 \%)$, and $16(30.8 \%)$ in Group 3, respectively $(p=0.050)$. The proportion of partici-
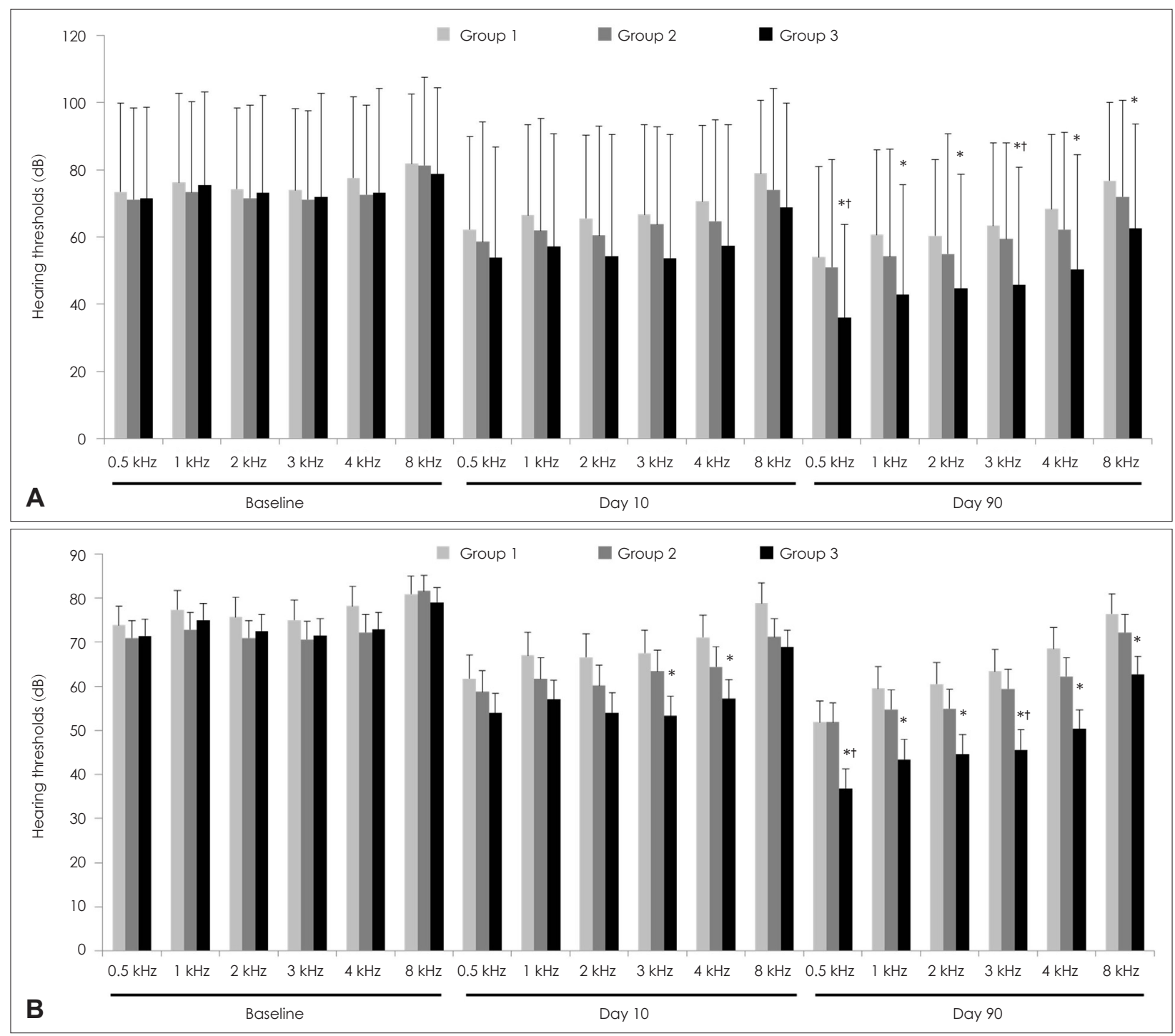

Fig. 1. Hearing thresholds according to groups. Univariate analysis (A). Multivariate analysis (B). The data are expressed as means \pm standard deviations in univariate analysis and means \pm standard errors in multivariate analysis. In univariate analyses, the $p$-values were tested using a one-way analysis of variance followed by post-hoc Turkey comparisons. In multivariate analyses, the $p$-values were tested using an analysis of covariance followed by an LSD post-hoc test. Multivariate analyses were adjusted for age and duration from onset to treatment. ${ }^{*} p<0.05$ vs. Group $1,+p<0.05$ vs. Group 2. 
pants with CR in Group 3 were significantly greater than that in the other groups ( $p=0.001)$. Logistic regression analysis revealed that Group 3 had greater odds ratio for CR compared with Group 1 (Table 2). Furthermore, Group 2 had greater odds ratio for CR compared with Group 1, although without any statistical significance.

The number of participants with CR in $<53$-years-old or $\geq 53$-years-old subgroups were $25(36.2 \%)$ and $8(11.3 \%)$, respectively $(p=0.001)$. Those whose duration from onset to treatment of $<3$ days or $\geq 3$ days were 13 (19.7\%) and $20(27 \%)$, respectively ( $p=0.308$ ). Those with diabetes or non-diabetes were $4(12.5 \%)$ and $29(26.9 \%)$, respectively ( $p=0.093)$. Those with and without hypertension were $3(15 \%)$ and 30 (25\%), respectively $(p=0.329)$. Those with AHT of $<70 \mathrm{~dB}$ or $\geq 70 \mathrm{~dB}$ were $25(40.3 \%)$ and $8(10.3 \%)$, respectively $(p<0.001)$. Those with and without tinnitus were $15(18.3 \%)$ and $18(31 \%)$, respectively $(p=0.080)$.

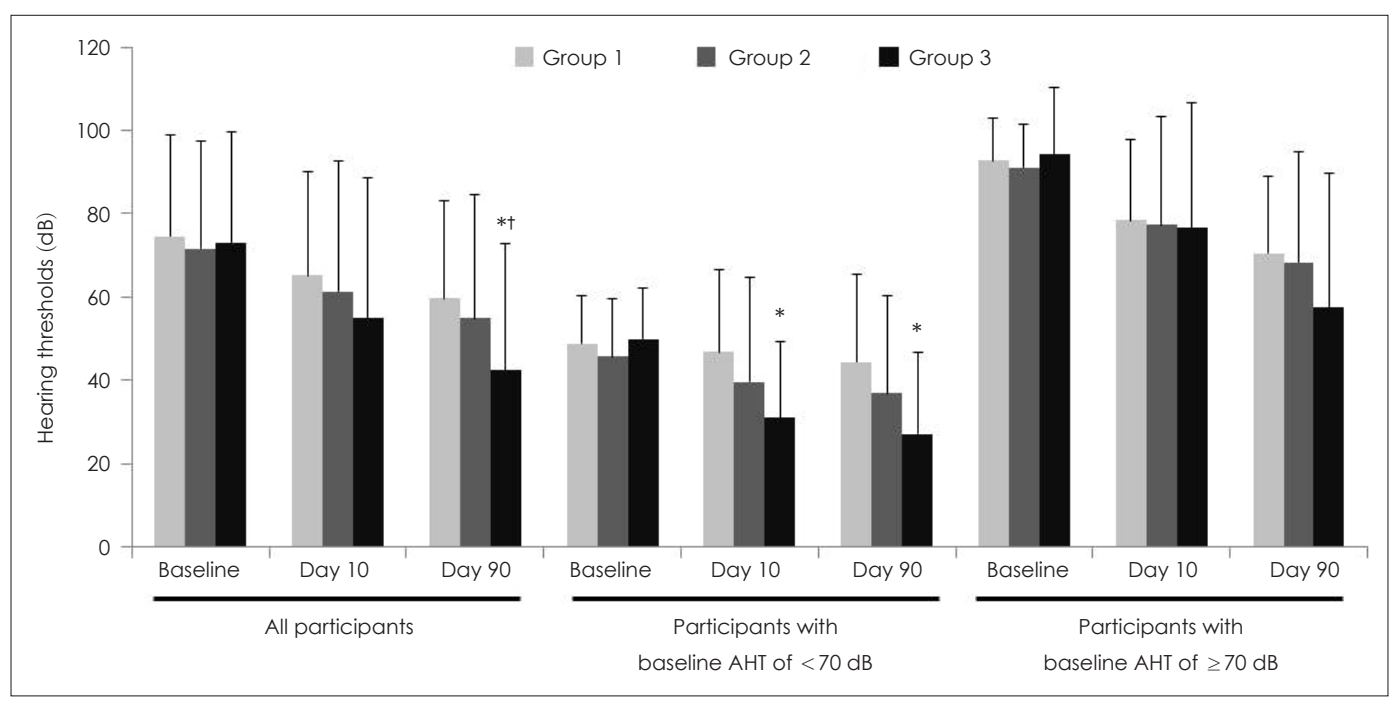

Fig. 2. Changes in AHT according to groups. Data were expressed as mean and standard deviation. For all participants, AHT in Groups 1,2 , and 3 were $74.5 \pm 24.4,71.6 \pm 25.7$, and $73.0 \pm 26.6 \mathrm{~dB}$ at baseline $(p=0.886) ; 65.2 \pm 25.0,61.2 \pm 31.5$, and $54.8 \pm 33.7 \mathrm{~dB}$ on Day 10 $(p=0.251)$; and $59.6 \pm 23.3,54.9 \pm 29.5$, and $42.3 \pm 30.5 \mathrm{~dB}$ on Day 90 , respectively $(p=0.013)$. For participants with a baseline AHT of $<70$ $\mathrm{dB}$, values in Groups 1,2 , and 3 were $48.7 \pm 11.4,45.6 \pm 14.0$, and $49.8 \pm 12.3 \mathrm{~dB}$ at baseline $(p=0.539) ; 46.7 \pm 20.0,39.5 \pm 25.0$, and $31.0 \pm 18.1 \mathrm{~dB}$ on Day $10(p=0.064)$; and $44.3 \pm 21.1,36.8 \pm 23.4$, and $27.1 \pm 19.4 \mathrm{~dB}$ on Day 90 , respectively $(p=0.044)$. For participants with a baseline AHT of $\geq 70 \mathrm{~dB}$, values in Groups 1,2 , and 3 were $92.7 \pm 10.2,91.1 \pm 10.3$, and $94.4 \pm 16.0 \mathrm{~dB}$ at baseline $(p=0.623) ; 78.4 \pm 19.3$, $77.3 \pm 25.9$, and $76.8 \pm 29.7 \mathrm{~dB}$ on Day $10(p=0.974)$; and $70.5 \pm 18.4,68.3 \pm 26.4$, and $57.5 \pm 32.2 \mathrm{~dB}$ on Day 90 , respectively $(p=0.198)$. Statistical analyses were performed using a one-way analysis of variance followed by post-hoc Turkey comparisons. Superscripts reveal statistical significance from post-hoc Turkey comparison while raw values without superscripts reveal non-statistical significance. ${ }^{*} p<0.05$ vs. the Group $1,+p<0.05$ vs. the Group 2. AHT: average hearing threshold.

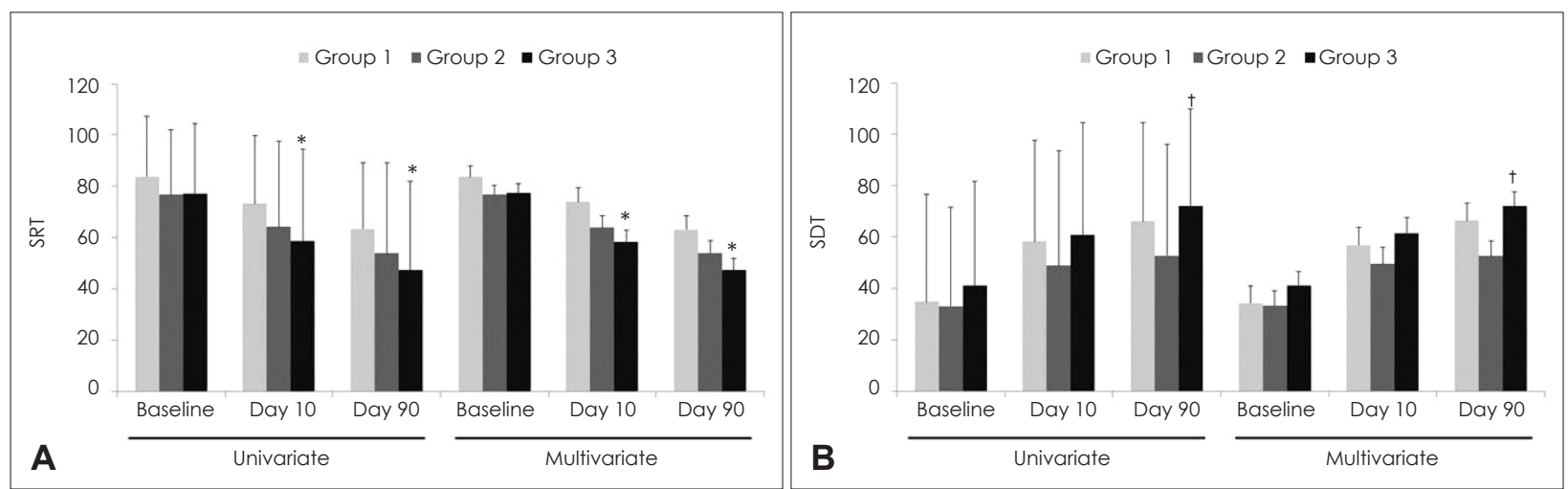

Fig. 3. Changes in SRT and SDT according to groups. SRT (A). SDT (B). Data were expressed as mean and standard deviation in univariate analysis and as mean and standard error in multivariate analysis. In univariate analyses, the $p$-values were tested using a oneway analysis of variance, followed by post-hoc Turkey comparisons. In multivariate analyses, the $p$-values were tested using an analysis of covariance followed by an LSD post-hoc test. Multivariate analyses were adjusted for age and duration from onset to treatment. Superscripts reveal statistical significance from post-hoc Turkey comparisons while raw values without superscripts reveal non-statistical significance. ${ }^{*} p<0.05$ vs. Group $1,+p<0.05$ vs. Group 2 . SRT: speech reception threshold, SDT: speech discrimination threshold. 
Table 2. Logistic regression for the odds ratio of CR according to groups

\begin{tabular}{|c|c|c|c|c|}
\hline & \multicolumn{2}{|c|}{ Univariate } & \multicolumn{2}{|c|}{ Multivariate } \\
\hline & OR $(95 \% \mathrm{Cl})$ & p-value & OR $(95 \% \mathrm{Cl})$ & p-value \\
\hline Age (increase 1 year) & $0.97(0.95-0.99)$ & 0.009 & $0.98(0.95-1.00)$ & 0.048 \\
\hline Duration from onset to treatment (increase 1 day) & $0.98(0.88-1.09)$ & 0.710 & $1.03(0.92-1.16)$ & 0.593 \\
\hline \multicolumn{5}{|l|}{ Group } \\
\hline \multicolumn{5}{|l|}{ Group 1 (ref) } \\
\hline Group 2 & $3.87(1.00-15.01)$ & 0.050 & $3.43(0.80-14.74)$ & 0.097 \\
\hline Group 3 & $7.29(1.98-26.87)$ & 0.003 & $6.62(1.63-26.87)$ & 0.008 \\
\hline \multicolumn{5}{|l|}{ Group 2 (ref) } \\
\hline Group 3 & $1.88(0.78-4.54)$ & 0.158 & $1.93(0.78-4.76)$ & 0.153 \\
\hline
\end{tabular}

Univariate analysis was performed using logistic regression analysis including one variable, while multivariate analysis was performed using logistic regression including age, duration from onset to treatment, and group. CR: complete remission, OR: odds ratio, $\mathrm{Cl}$ : confidence interval

\section{Discussion}

Our data of both univariate and multivariate analyses shows that Group 3 had the lowest hearing thresholds. The impact of favorable thresholds in Group 3 was better among patients with a baseline AHT of $<70 \mathrm{~dB}$. For patients with a baseline $\mathrm{AHT} \geq 70 \mathrm{~dB}$, the results showed a similar trend, but with weak statistical significance. Group 3 also showed the most favorable results for SRT and SDS. In addition, Group 3 had a greater odds ratio for CR compared with Group 1 and/or Group 2.

Sensorineural hearing loss can result from definite causes such as malignancy, infection, or metabolic and vascular problems, but most cases are idiopathic. Steroid therapy can be effective in cases of viral, vascular, or immunologic etiology and in endolymphatic hydrops. ${ }^{2,10)}$ A previous study showed that an inflammatory response is associated with ISSNHL and that the inflammation can be modified by steroid therapy. ${ }^{11)}$ Wilson, et al. ${ }^{12)}$ reported that hearing improved after treatment with systemic steroids compared to placebo, after which many other studies have evaluated the efficacy of steroids in ISSNHL. However, recent meta-analysis showed negative results regarding the effect of steroids. ${ }^{13,14)}$ Althouth the risk-to-benefit ratio and the efficacy of steroids remain obscure, some large observational studies showed beneficial effects of its use in ISSNHL. Therefore, recent clinical guidelines suggest that steroid therapy within two weeks of symptom onset could be an option for the treatment of ISSNHL." However, uncertainties over the efficacy of systemic steroid remain, with IT steroid injection being the more commonly used option for ISSNHL due to its theoretical advantage compared to systemic steroids. ${ }^{15-17)}$ Many studies showed favor- able results with the use of combined treatment with systemic and IT steroids. ${ }^{18,19)}$

Superiority of Group 3 over Group 1 is a prominent result in the present study. Some factors may be associated with this result as follows: differences in admission period, duration of steroid activity, and steroid dose. First, a longer admission period may be associated with favorable results. A previous study showed that the recovery rate of out-patient department-based treatment was greater than that of admission based treatment ${ }^{20)}$ during hospitalization, patients are more likely to required bed-rest, consume a low-salt diet, remain hydrated, and be free of social work and psychologic stress, which could lead to favorable results of admissionbased treatment. Our results may be an extension of such findings from that previous study. Second, difference in steroid regimen may be associated with favorable results. Dexamethasone has a longer duration of action compared with prednisolone. ${ }^{19)}$ Duration of action of prednisolone and dexamethasone was $12-36$ and 36-72 h, respectively. Although the total duration of steroid was similar, long-acting steroids may be associated with greater cumulative dose and longer effect of steroid that those associated with short-acting steroids. Third, there was a difference in total steroid dose. Group 1 and Group 3 used an approximate dexamethasonebased dose of $69.5 \mathrm{mg}$ and $75 \mathrm{mg}$, respectively. A difference of $5.5 \mathrm{mg}$ between these dexamethasone doses (approximate dose per day) could influence the outcome. IT steroid was administered thrice in both Group 1 and Group 3, but IT steroid injection alone would not lead to a difference in outcomes between the two groups.

Although statistical significances were not obtained, there was a modest difference in outcome between Group 2 and 
Group 1 or Group 3. Group 2 had little favorable outcomes compared with Group 1. Total dexamethasone-based steroid dose in Group 1 and Group 2 was 69.5 and $75 \mathrm{mg}$, respectively. In addition, admission period was longer in Group 2 than in Group 1, and steroid regimen may be more effective in Group 2 than in Group 1. However, IT steroid injection was administered in Group 1 alone. Favorable factors in Group 2, such as longer admission period and steroid regimen, would offset the impact of systemic steroid alone without the need for IT steroid administration. Systemic steroid dose was the same between Group 2 and Group 3, but IT steroid injection was administered in Group 3 alone. Group 3 showed a modest improvement compared with Group 2, which could be attributed to the addition of IT steroid injection in Group 3.

We hypothesized an association between systemic steroid dose and hearing for the interpretation of our results. The three steroid regimens in our study were different, but it is unclear whether differences in these regimens would lead to significant differences in clinical outcomes. The absence of IT steroid injection in Group 2 may be associated with a greater clinical impact rather than the difference in systemic steroid dose between groups. However, in our study, the difference in hearing outcomes between Group 1 and Group 3 was greatest. This may be due to uncontrolled confounding factors such as age, duration from onset to treatment, tinnitus, or additional therapies in spite of multivariate analyses. Randomized controlled trials involving larger groups with different steroid regimens are needed to overcome differences in baseline variables and the presence of additional therapies. Despite its limitations, our study provided some perspective on the beneficial effects of different steroid regimens for improved hearing.

This study had a few limitations. First, it had a retrospective design and based on data from a single center. The treatment method was divided according to the changes in treatment protocols of our hospital. This could have led to selection bias. Second, the patient sample size was small. Third, our study did not include long-term follow up data. Fourth, baseline age and duration from onset to treatment were different among the three groups in our study. These would be confounding factors and/or prognostic factors for hearing recovery but they can be overcome by propensity score matching and/or multivariate analysis. We did not perform propensity score matching due to the small sample size. However, we performed multivariate analyses, adjust- ing age and duration from onset to treatment, and subgroup analysis by baseline AHT level. These results were similar with those from univariate analyses. Fifth, our study was of the retrospective type, which used medical chart reviews and did not completely exclude additional therapies during follow-up. Additional therapies, such as herbal medication and/ or steroids, would be considered as confounding factors. However, in our study, all patients were followed up from initial therapy until day 90 in our hospital. None of the patients underwent additional therapies in our hospital and no patient wanted to transfer to other hospital. Furthermore, none of them reported that they were undergoing any additional therapies.

In conclusion, administration of dexamethasone-based systemic steroid combined with IT steroid injection and a relatively long hospitalization period produced the most favorable result. Further randomized controlled studies that include long-term follow-up data are warranted to overcome selection bias and evaluate a possible correlation between clinical outcome and steroid regimen, IT steroid injection, or admission period.

\section{Supplementary Materials}

The Data Supplement is available with this article at https://doi. org/10.3342/kjorl-hns.2020.00066.

\section{Acknowledgments}

This work was supported by the National Research Foundation of Korea (NRF) grant funded by the Korea government (MSIT) (2018R1C1B6007775).

\section{Author Contribution}

Conceptualization: Da Jung Jung. Data curation: JiHoon Lee. Formal analysis: Da Jung Jung. Funding acquisition: Da Jung Jung. Investigation: Myung Hoon Yoo. Methodology: Kyu-Yup Lee. Project administration: Da Jung Jung, Kyu-Yup Lee. Resources: Da Jung Jung, Myung Hoon Yoo, Kyu-Yup Lee. Software: Da Jung Jung. Supervision: Kyu-Yup Lee. Validation: JiHoon Lee. Visualization: Da Jung Jung. Writing — original draft: Da Jung Jung.

\section{ORCIDs}

Kyu-Yup Lee https://orcid.org/0000-0001-7170-4847

Da Jung Jung https://orcid.org/0000-0001-6178-6113

Yoo Myung Hoon https://orcid.org/0000-0002-9158-8924

\section{REFERENCES}

1) Byl FM. Seventy-six cases of presumed sudden hearing loss occurring in 1973: Prognosis and incidence. Laryngoscope 1977; 87(5 Pt 1):817-25.

2) Mattox DE, Simmons FB. Natural history of sudden sensorineural hearing loss. Ann Otol Rhinol Laryngol 1977;86(4 Pt 1):463-80.

3) Alexander TH, Harris JP. Incidence of sudden sensorineural 
hearing loss. Otol Neurotol 2013;34(9):1586-9.

4) Jung SY, Shim HS, Hah YM, Kim SH, Yeo SG. Association of metabolic syndrome with sudden sensorineural hearing loss. JAMA Otolaryngol Head Neck Surg 2018;144(4):308-14.

5) Schreiber BE, Agrup C, Haskard DO, Luxon LM. Sudden sensorineural hearing loss. Lancet 2010;375(9721):1203-11.

6) Crane RA, Camilon M, Nguyen S, Meyer TA. Steroids for treatment of sudden sensorineural hearing loss: A meta-analysis of randomized controlled trials. Laryngoscope 2015;125(1):209-17.

7) Chandrasekhar SS, Tsai Do BS, Schwartz SR, Bontempo LJ, Faucett EA, Finestone SA, et al. Clinical practice guideline: Sudden hearing loss (update). Otolaryngol Head Neck Surg 2019; 161(1_suppl):S1-45.

8) Gao Y, Liu D. Combined intratympanic and systemic use of steroids for idiopathic sudden sensorineural hearing loss: A metaanalysis. Eur Arch Otorhinolaryngol 2016;273(11):3699-711.

9) Siegel LG. The treatment of idiopathic sudden sensorineural hearing loss. Otolaryngol Clin North Am 1975;8(2):467-73.

10) Norris CH. Drugs affecting the inner ear. A review of their clinical efficacy, mechanisms of action, toxicity, and place in therapy. Drugs 1988;36(6):754-72.

11) Hiramatsu M, Teranishi M, Uchida Y, Nishio N, Suzuki H, Kato K, et al. Polymorphisms in genes involved in inflammatory pathways in patients with sudden sensorineural hearing loss. J Neurogenet 2012;26(3-4):387-96.

12) Wilson WR, Byl FM, Laird N. The efficacy of steroids in the treatment of idiopathic sudden hearing loss. A double-blind clinical study. Arch Otolaryngol 1980;106(12):772-6

13) Wei BP, Stathopoulos D, O'Leary S. Steroids for idiopathic sudden sensorineural hearing loss. Cochrane Database Syst Rev 2013;(7): CD003998

14) Mirian $C$, Ovesen $T$. Intratympanic vs systemic corticosteroids in first-line treatment of idiopathic sudden sensorineural hearing loss: A systematic review and meta-analysis. JAMA Otolaryngol Head Neck Surg 2020;146(5):1-8.

15) Nosrati-Zarenoe R, Hultcrantz E. Corticosteroid treatment of idiopathic sudden sensorineural hearing loss: Randomized tripleblind placebo-controlled trial. Otol Neurotol 2012;33(4):523-31.

16) Cinamon U, Bendet E, Kronenberg J. Steroids, carbogen or placebo for sudden hearing loss: A prospective double-blind study. Eur Arch Otorhinolaryngol 2001;258(9):477-80.

17) Parnes LS, Sun AH, Freeman DJ. Corticosteroid pharmacokinetics in the inner ear fluids: An animal study followed by clinical application. Laryngoscope 1999;109(7 Pt 2):1-17.

18) Jung da J, Park JH, Jang JH, Lee KY. The efficacy of combination therapy for idiopathic sudden sensorineural hearing loss. Laryngoscope 2016;126(8):1871-6.

19) Li J, Ding L. Effectiveness of steroid treatment for sudden sensorineural hearing loss: A meta-analysis of randomized controlled trials. Ann Pharmacother 2020;54(10):949-57.

20) Kim MJ, Heo KW, Jeon PH, Lee JJ, Lee JH. Necessity of admission to improve the hearing-recovery rate in idiopathic sudden sensorineural hearing loss. Acta Otolaryngol 2018;138(4):357-62. 


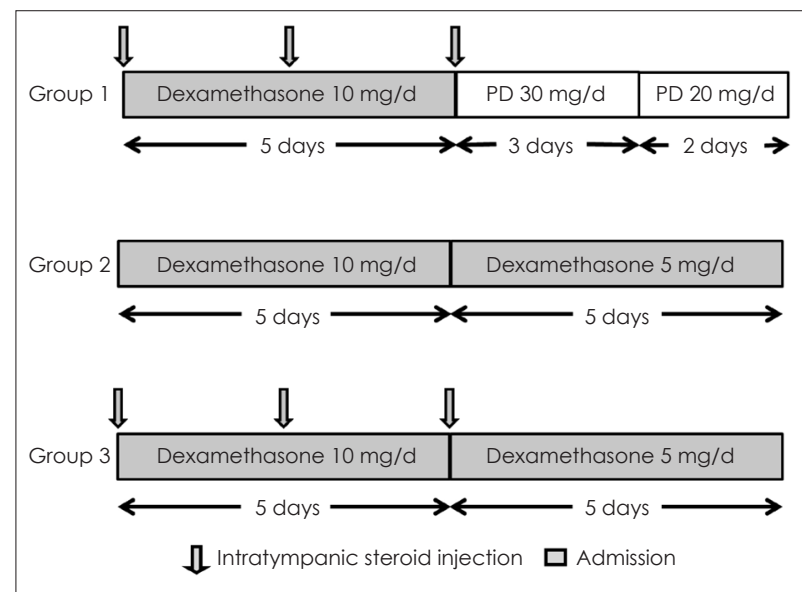

Supplementary Fig. 1. Schematic protocol according to three groups. PD: prednisolone. 
Supplementary Table 1. Hearing thresholds according to groups

\begin{tabular}{|c|c|c|c|c|c|c|c|c|}
\hline & \multicolumn{4}{|c|}{ Univariate } & \multicolumn{4}{|c|}{ Multivariate } \\
\hline & Group 1 & Group 2 & Group 3 & $p$-value & Group 1 & Group 2 & Group 3 & $p$-value \\
\hline \multicolumn{9}{|l|}{ Baselines } \\
\hline $0.5 \mathrm{kHz}$ & $73.4 \pm 26.4$ & $71.1 \pm 27.2$ & $71.5 \pm 27.0$ & 0.912 & $73.8 \pm 4.4$ & $70.9 \pm 4.0$ & $71.4 \pm 3.8$ & 0.885 \\
\hline $1 \mathrm{kHz}$ & $76.2 \pm 26.5$ & $73.3 \pm 26.9$ & $75.4 \pm 27.7$ & 0.870 & $77.3 \pm 4.4$ & $72.8 \pm 4.0$ & $75.0 \pm 3.8$ & 0.763 \\
\hline $2 \mathrm{kHz}$ & $74.3 \pm 24.1$ & $71.6 \pm 27.6$ & $73.1 \pm 29.0$ & 0.898 & $75.7 \pm 4.5$ & $70.9 \pm 4.0$ & $72.5 \pm 3.8$ & 0.737 \\
\hline $3 \mathrm{kHz}$ & $73.9 \pm 24.3$ & $71.1 \pm 26.4$ & $71.9 \pm 30.9$ & 0.887 & $75.0 \pm 4.5$ & $70.6 \pm 4.1$ & $71.5 \pm 3.9$ & 0.767 \\
\hline $4 \mathrm{kHz}$ & $77.6 \pm 24.0$ & $72.6 \pm 26.5$ & $73.2 \pm 31.0$ & 0.656 & $78.2 \pm 4.5$ & $72.2 \pm 4.0$ & $72.9 \pm 3.8$ & 0.589 \\
\hline $8 \mathrm{kHz}$ & $81.8 \pm 20.7$ & $81.3 \pm 26.1$ & $78.7 \pm 25.7$ & 0.793 & $80.9 \pm 4.0$ & $81.6 \pm 3.5$ & $79.0 \pm 3.4$ & 0.858 \\
\hline \multicolumn{9}{|l|}{ Day 10} \\
\hline $0.5 \mathrm{kHz}$ & $62.2 \pm 27.6$ & $58.6 \pm 35.6$ & $53.8 \pm 33.0$ & 0.454 & $61.8 \pm 5.3$ & $58.7 \pm 4.8$ & $53.9 \pm 4.5$ & 0.523 \\
\hline $1 \mathrm{kHz}$ & $66.5 \pm 26.8$ & $61.9 \pm 33.3$ & $57.2 \pm 33.4$ & 0.374 & $67.0 \pm 5.2$ & $61.7 \pm 4.7$ & $57.0 \pm 4.4$ & 0.361 \\
\hline $2 \mathrm{kHz}$ & $65.6 \pm 24.7$ & $60.5 \pm 32.4$ & $54.4 \pm 36.0$ & 0.241 & $66.6 \pm 5.3$ & $60.1 \pm 4.7$ & $54.0 \pm 4.5$ & 0.203 \\
\hline $3 \mathrm{kHz}$ & $66.7 \pm 26.6$ & $63.8 \pm 29.0$ & $53.7 \pm 36.7$ & 0.107 & $67.5 \pm 5.2$ & $63.4 \pm 4.7$ & $53.3 \pm 4.4^{*}$ & 0.097 \\
\hline $4 \mathrm{kHz}$ & $70.6 \pm 22.6$ & $64.7 \pm 30.2$ & $57.4 \pm 35.9$ & 0.117 & $71.1 \pm 5.0$ & $64.4 \pm 4.5$ & $57.2 \pm 4.3^{*}$ & 0.117 \\
\hline $8 \mathrm{kHz}$ & $78.9 \pm 21.7$ & $74.1 \pm 30.0$ & $68.8 \pm 31.1$ & 0.227 & $78.8 \pm 4.6$ & $71.2 \pm 4.1$ & $68.8 \pm 3.9$ & 0.265 \\
\hline \multicolumn{9}{|l|}{ Day 90} \\
\hline $0.5 \mathrm{kHz}$ & $54.0 \pm 27.0$ & $51.0 \pm 32.1$ & $36.0 \pm 27.8^{*+}$ & 0.009 & $51.9 \pm 4.8$ & $51.9 \pm 4.3$ & $36.9 \pm 4.3^{*+}$ & 0.022 \\
\hline $1 \mathrm{kHz}$ & $60.7 \pm 25.3$ & $54.3 \pm 31.8$ & $42.8 \pm 32.8^{*}$ & 0.022 & $59.5 \pm 5.0$ & $54.8 \pm 4.4$ & $43.4 \pm 4.5^{*}$ & 0.048 \\
\hline $2 \mathrm{kHz}$ & $60.4 \pm 22.6$ & $55.0 \pm 35.6$ & $44.7 \pm 34.0^{*}$ & 0.045 & $60.5 \pm 4.9$ & $54.9 \pm 4.4$ & $44.6 \pm 4.4^{*}$ & 0.054 \\
\hline $3 \mathrm{kHz}$ & $63.4 \pm 24.7$ & $59.4 \pm 28.6$ & $45.7 \pm 35.0^{*+}$ & 0.015 & $63.4 \pm 5.0$ & $59.4 \pm 4.4$ & $45.6 \pm 4.5^{* \dagger}$ & 0.020 \\
\hline $4 \mathrm{kHz}$ & $68.4 \pm 22.1$ & $62.2 \pm 28.9$ & $50.3 \pm 34.1^{*}$ & 0.014 & $68.5 \pm 4.8$ & $62.2 \pm 4.3$ & $50.3 \pm 4.3^{*}$ & 0.018 \\
\hline $8 \mathrm{kHz}$ & $76.8 \pm 23.3$ & $72.0 \pm 28.7$ & $62.5 \pm 31.1^{*}$ & 0.054 & $76.4 \pm 4.5$ & $72.2 \pm 4.1$ & $62.7 \pm 4.1^{*}$ & 0.072 \\
\hline
\end{tabular}

The data are expressed as means \pm standard deviations in univariate analysis and means \pm standard errors in multivariate analysis. In univariate and multivariate analyses, the p-values were tested using a one-way analysis of variance followed by post-hoc Turkey comparisons, and an analysis of covariance followed by an LSD post-hoc test, respectively. Multivariate analyses were adjusted for age and duration from onset to treatment. ${ }^{*} p<0.05$ vs. Group $1,{ }^{t} p<0.05$ vs. Group 2 